\title{
Photopolymerization monitoring of ceramic stereolithography resins by FTIR methods
}

\author{
K. C. WU, J. W. HALLORAN \\ Department of Materials Science and Engineering, University of Michigan, \\ Ann Arbor, MI 48109, USA \\ E-mail: john_halloran@engin.umich.edu
}

Curing kinetics of UV photocurable acrylate monomer-based ceramic resins were studied using a Fourier Transform Infrared (FTIR) spectroscopy instrument with an internally-mounted UV-curing source, providing real-time quantification of the acrylate double bond conversion with respect to increasing UV dose. The results show that this Real Time FTIR experiment (RTFTIR) provides a simple but valuable means of investigating average photopolymerization kinetics and final conversion in a thin layer of highly loaded ceramic suspension. This RTFTIR technique showed complete agreement with differential photo-calorimetry (photoDSC) experiments performed on the same thickness and amount highly loaded ceramic suspension.

FTIR was also used to investigate the curing behavior inside sublayers of the same ceramic suspension. Curing kinetics and final conversion were monitored in these layers, with higher conversion and faster kinetics being observed near the exposed surface. Due to the scattering and absorption effects within the uppermost layers, photopolymerization rate and percent conversion declined as a function of depth from the surface.

(C) 2005 Springer Science + Business Media, Inc.

\section{Introduction}

Interest in UV curing systems has grown rapidly in the recent decades among coatings and ink researchers due to the fact that these systems offer several advantages over thermal curing, including instant cure, no volatile organic solvents, and ease of automation [1]. The strong commercial demand, coupled by the development of high power and variable wavelength UV lamps, have increased the availability of novel monomers, oligomers, photoinitiators for UV curable formulations. These formulations have found a wide spectrum of applications, and have become an integral part of ceramic stereolithography [2-5].

In the coatings and ink industry, photopolymerization reaction kinetics of formulations are often monitored pre-cure and post-cure by Fourier transform infrared spectroscopy (FTIR) to determine a final percent conversion of the monomer. FTIR is also used to determine the chemical structures of the polymeric samples, deduced by the types of organic bonding evident in the resultant infrared absorption spectrum. Following on another real time method, photoDSC, this experiment aims to apply the FTIR to the determination of photopolymerization rate kinetics, thus adapting an intrinsically qualitative analytical tool into a quantitative one. The photoDSC technique has been well established, and serves to confirm the results of this experiment.

In an FTIR experiment, a sample is radiated in the infrared region of the electromagnetic spectrum. As the infrared range of interest is scanned, the molecular structure affects the infrared absorption at certain wavelengths, namely due to bond stretching or twisting motions. These absorption peaks on the resultant spectra can then be identified with known spectra of known compounds. In this experiment, the target molecular structure to be monitored is the acrylate carbon double bond. Although FTIR is primarily used to qualitatively 'see' which bond structures are present within a sample, the absorption peak areas are proportional to the concentration of the bond structures, allowing for quantifiable comparisons. Operating principles of the FTIR can be found elsewhere.

However, most photopolymeric studies in FTIR and photoDSC have been performed on clear monomers and oligomers. In ceramic stereolithography, suspensions are formulated with almost the same monomers and oligomers commonly found in UV curable clear coatings, but the addition of ceramic fillers, especially at loadings of $50 \mathrm{vol} \%$, complicates curing behavior due to competition for UV light absorption, light scattering, and even surface chemistry [6]. Over the years, most work done to investigate UV curing behavior of highly filled suspensions focused on qualitative testing methods including surface scratch, adhesion, and solvent resistance [7]. The experiments presented here aim to apply FTIR techniques to characterize photopolymerization reactions of ceramic stereolithography resins 
and to investigate the effect of the added fillers on the curing behavior.

Knowledge of the photopolymerization reaction is essential in determining the proper UV dose necessary for ceramic stereolithography. The dose required to photopolymerize a pane of ceramic suspension can also be roughly compared to the results shown by FTIR, and the corresponding percent conversion of the monomer at the minimum dose required should be beyond the critical 'gel point', at which the ceramic particles and the monomer molecules begin to immobilize as a result of their polymerizing environment $[8,9]$.

The photopolymerizable ceramic-acrylate systems created in the Griffith, Brady, and Chu experiments showed a wide range of applicable monomers and photoinitiators, and combinations thereof $[6,11,12]$. Furthermore, as other classes of monomers are added to suspension recipes, such as cationic polymerizing species, the photoDSC technique may not be suitable due to the fact that exotherms result from both photoinitiation and a number of polymerization pathways different from carbon double bond conversion [10]. The application of FTIR in characterizing these suspensions seems promising, but must be validated first.

\section{Experimental description}

\subsection{Materials}

This study focused on the 1,6-hexanediol diacrylate (HDDA) monomer, shown in Fig. 1, a difunctional monomer of low viscosity and low refractive index, and density of $1200 \mathrm{~kg} / \mathrm{m}^{3}$. Alumina filler (A16SG, 0.4 micron mean diameter) was obtained from $\mathrm{Al}-$ coa, and stored in a drying oven prior to use to minimize moisture-induced flocculation in suspension. The photoinitiators, a phenylphosphineoxide (PPO) and a hydroxy-ketone (HK) were used as received from Ciba Specialty Chemicals, and are both very soluble in the monomer HDDA. Chemical structures and UV absorption characteristics of the photoinitiators are shown in Fig. 2.

\subsection{Photopolymerizable alumina-HDDA suspension at $50 \mathrm{vol} \%$ loading}

The ceramic suspension was made by incrementally adding the alumina powder to the HDDA monomer in amounts that resulted in a $50 \mathrm{vol} \%$ alumina suspen-

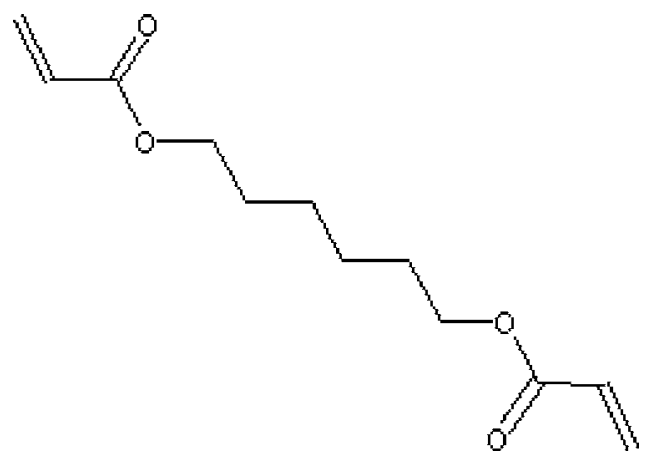

Figure 1 1,6-Hexandiol diacrylate (HDDA) monomer.

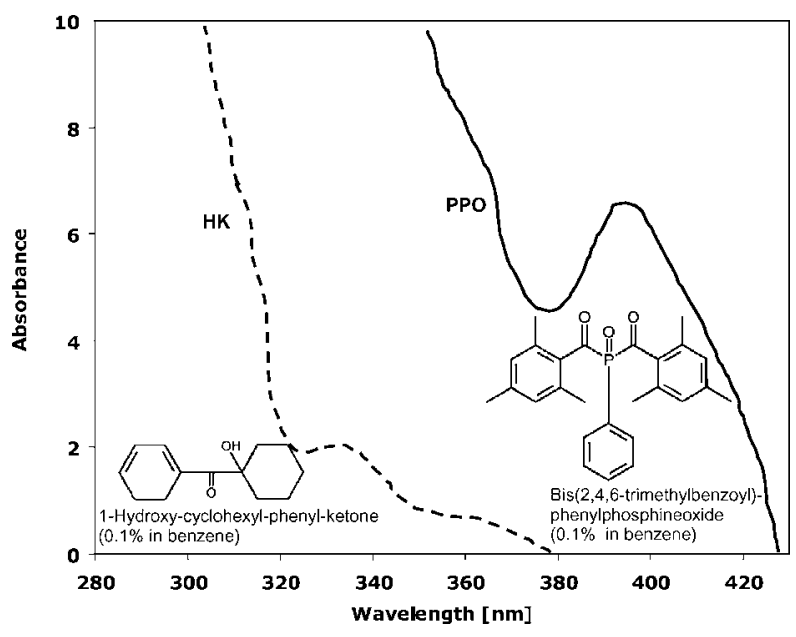

Figure 2 Photoinitiator species and absorption properties.

sion. A cationic quaternary amine dispersant, CC-59, obtained from Goldschmidt, was added at $2 \%$ of the solids weight to lower the viscosity and aid in ceramic dispersion. The mixture was ball milled with $6.35 \mathrm{~mm}$ diameter alumina round milling media for $48 \mathrm{~h}$, yielding an easily pouring ceramic suspension with a viscosity of about $0.26 \mathrm{~Pa} \cdot \mathrm{s}$, as shown in Fig. 3. The suspension shows some structure, which is broken down at the low shear rates to yield a nearly Newtonian fluid at intermediate shear rates, indicating good stability and colloidal dispersion.

Using this recipe, separate suspensions were made with the two types of photoinitiators at varying concentrations. The compositions of the three photocurable ceramic suspensions studied in this experiment, labeled as A, B, and C, are described in Table I. As a control, the experiments were also performed with unfilled monomer, labeled as D.

\subsection{Real-time FTIR setup}

A Perkin-Elmer FTIR, in transmission mode, was used for this study on films of up to 300 microns of the photocurable alumina/HDDA suspensions discussed above. A small amount $(\sim 5 \mathrm{~g})$ of the resin was placed

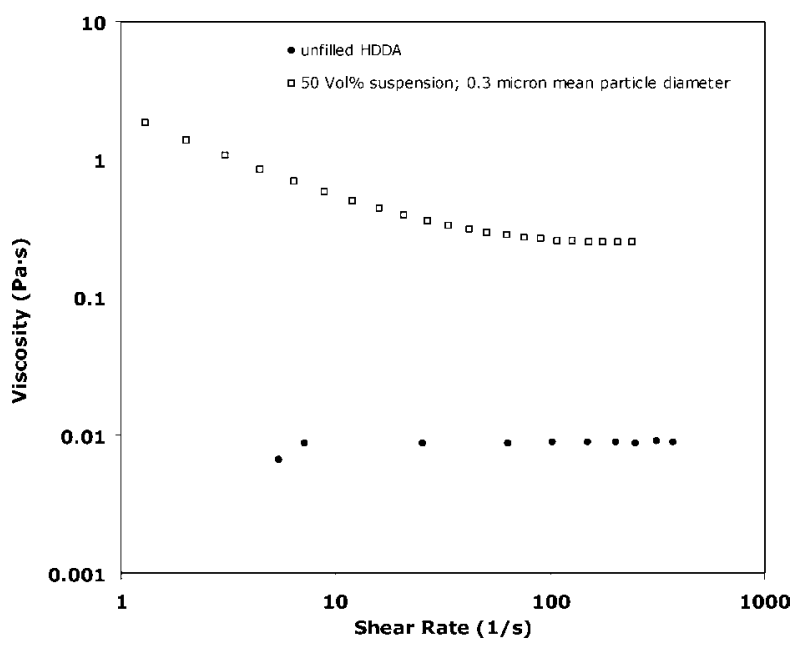

Figure 3 Viscosity behavior of alumina suspension in HDDA. 
TABLE I Experimental ceramic suspensions.

\begin{tabular}{llll}
\hline & A & B & C \\
\hline $\begin{array}{l}\text { Ceramic: } \\
(50 \text { vol } \%)\end{array}$ & $\begin{array}{c}\text { Alcoa A16SG, } \\
\text { mean particle } \\
\text { diameter }=0.4 \mu \mathrm{m}\end{array}$ & $\begin{array}{c}\text { Alcoa A16SG, } \\
\text { mean particle } \\
\text { diameter }=0.4 \mu \mathrm{m}\end{array}$ & $\begin{array}{c}\text { Alcoa A16SG, } \\
\text { mean particle } \\
\text { diameter }=0.4 \mu \mathrm{m}\end{array}$ \\
$\begin{array}{l}\text { Dispersant: } \\
(2 \text { solids wt })\end{array}$ & Cationic & Cationic & Cationic \\
$\begin{array}{l}\text { Monomer: } \\
\begin{array}{c}\text { Photoinitiator: } \\
(\text { monomer } w \mathrm{w} \%)\end{array}\end{array}$ & HDDA & Quaternary Amine & Quaternary Amine \\
\hline
\end{tabular}

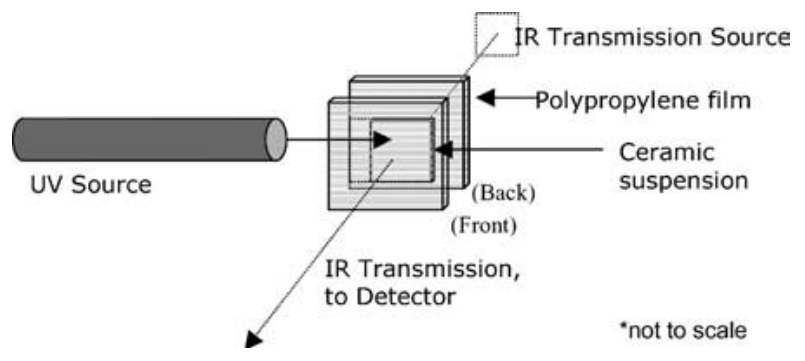

Figure 4 RTFTIR experimental diagram.

between two sheets of polypropylene film (6 micron thickness, SPEX Certiprep, Fisher Scientific), where capillary forces maintained a thin film of approximately 150 microns, measured post-cure with a micrometer. The sample was positioned in front of the IR beam source, and a UV source (UVP Light-pen, peak emission at $320 \mathrm{~nm}$ ) was positioned a distance of approximately $30 \mathrm{~mm}$ from the sample, without obstructing the IR beam path to the detector (Fig. 4). The intensity of the UV source at the exact position of the sample being analyzed was determined to be $140 \mathrm{~W} / \mathrm{m}^{2}$ in the wavelength range of $308-360 \mathrm{~nm}$ using a radiometer (UVX, UVP Inc.). The polypropylene film material showed $99.8 \%$ transmittance of the UV light intensity and has negligible infrared absorption spectra with regard to the sample of interest. The machine is operated through Perkin-Elmer FTIR Spectrum Analysis software, where FTIR transmission scans were repeatedly recorded at $4 \mathrm{~cm}^{-1}$ resolution after the UV source was turned on (time $t=0$ ) to follow the acrylate double bond conversion in real time.

\subsection{Differential photo calorimetry}

Differential photo calorimetry, which has been established as a reliable and quantifiable means of monitoring photopolymerization reactions, was used to verify the results shown in the FTIR experiments. A PerkinElmer DSC7 unit without purge gas was used for this experiment, for which a modified sample cover was customized to accommodate a UV light source with two quartz windows directly positioned over the sample and reference cells, as shown in Fig. 5. The intensity at the distance of the sample and reference cells was measured to be $140 \mathrm{~W} / \mathrm{m}^{2}$ in the wavelength range of 308-360 nm. Approximately $18 \mathrm{mg}$ of photocurable ceramic suspensions under study were placed in the shallow depression on the aluminum sample pan lids.

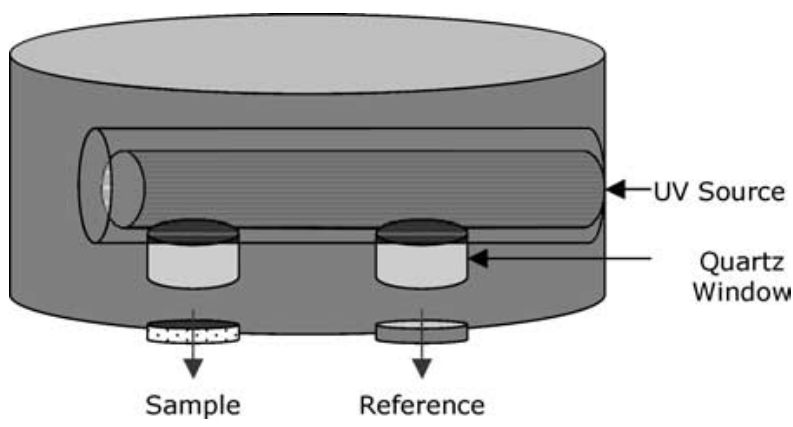

Figure 5 PhotoDSC customized head mounted on perkin elmer DSC7.

The minimal wall height around the sample prevented the suspension from gelling to the sides and leaving the center of the pan empty, allowing for a thin film of suspension to be maintained during the experiment. The experiments were performed for approximately $10 \mathrm{~min}$, or the equivalent dose of about $90 \mathrm{~kJ} / \mathrm{m}^{2}$. The data reported for each suspension is an average of three separate photopolymerizations.

\subsection{Layered FTIR experiment}

The photopolymerizable ceramic suspensions were placed between polypropylene films in thicknesses of approximately 80 microns. Three of these polypropylene-suspension-polypropylene sandwiched layers were assembled together, giving a total stack thickness (each stack consisting of the series film-sample-film-film-sample-film-film-samplefilm) of approximately 250 microns (Fig. 6), with good reproducibility. While using the same FTIR setup as described above, this experiment was not carried out in real-time. Each assembled stack was exposed to increasing amounts of UV exposure dose on the topside. Each stack was then disassembled, and FTIR scans of

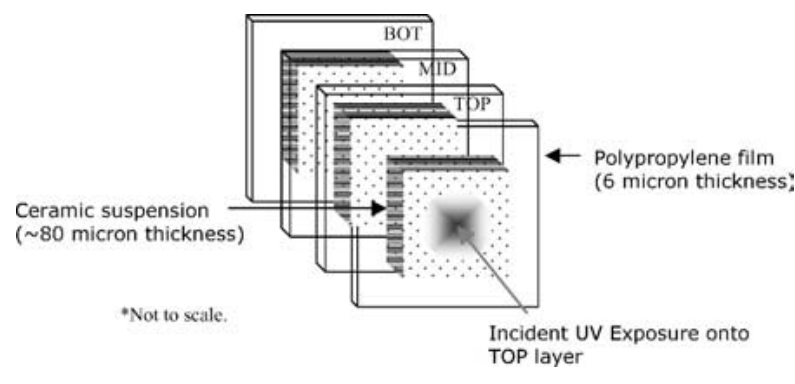

Figure 6 Layered FTIR sample diagram. 


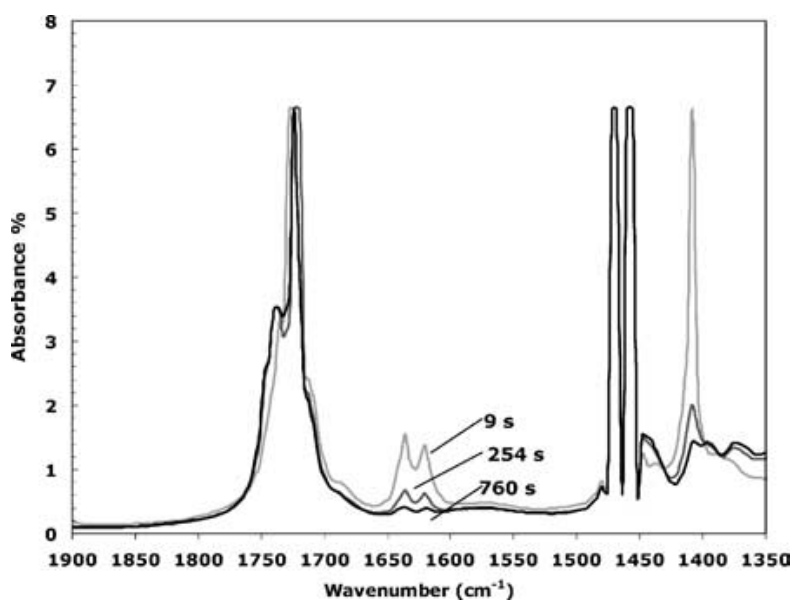

Figure 7 FTIR scans showing photopolymerization with time.

each layer were performed. This allowed for the observation of curing behavior as a function of layer thickness and exposure dose.

\section{Results}

\subsection{Real time FTIR (RTFTIR)}

An example real time FTIR scan in a sample range of interest of the alumina-acrylate suspension is shown in Fig. 7. The curing profile was monitored by following the carbon-carbon double bond regions of the acrylate structure, namely in the $1600-1660 \mathrm{~cm}^{-1}$ region, characteristic of $\mathrm{C}=\mathrm{C}$ stretching. Curing was also monitored in the $1400-1430 \mathrm{~cm}^{-1}$ region, characteristic of $\mathrm{C}=\mathrm{C}$ twisting. With increasing exposure dose (dose $=\mathrm{UV}$ source intensity $*$ time), these absorption bands were observed to decrease, indicating a decrease in double bond conversion. During the photopolymerization reaction, the main peak in the $1750 \mathrm{~cm}^{-1}$ region, which corresponds to the $\mathrm{C}=\mathrm{O}$ stretching absorption, is unchanged, providing for an internal reference for quantification of the $\mathrm{C}=\mathrm{C}$ bond peak area decline.

The percent conversion $(\alpha)$ of the acrylate was calculated using:

$$
\alpha(t)=\frac{\left[\frac{\text { Area }_{1620}+\text { Area }_{1410}}{\text { Area }_{1750}}\right]_{0}-\left[\frac{\text { Area }_{1620}+\text { Area }_{1410}}{\text { Area }_{1750}}\right]_{t}}{\left[\frac{\text { Area }_{1620}+\text { Area }_{1410}}{\text { Area }_{1750}}\right]_{0}} \times 100
$$

with peak areas noted by their maximum height in subscript. The presented results show conversion as a function of UV exposure dose, which is simply the measured intensity of the UV source $\left(\mathrm{W} / \mathrm{m}^{2}\right)$ multiplied by the time (seconds).

\subsection{PhotoDSC:}

The photoDSC device, which measures the exothermic heat flow from the free-radical photopolymerizing sample during irradiation, is a proven method of determining polymerization kinetics. With the acrylate monomer $\mathrm{C}=\mathrm{C}$ bond breakage as the only heat source during the experiment, the heat flow values could be calculated into percent conversion of monomer $\mathrm{C}=\mathrm{C}$ bonds. The

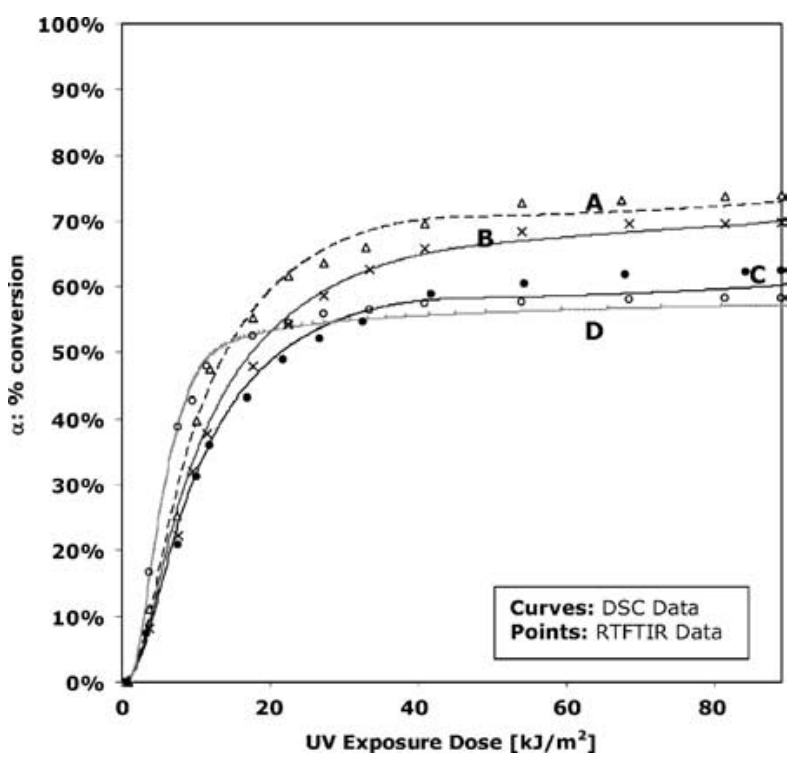

Figure 8 Percent conversion vs. exposure dose.

photopolymerization rate is determined from the measured heat flow by:

$$
R_{\mathrm{P}}=\frac{\mathrm{d} H}{\mathrm{~d} t} \cdot \frac{1}{\Delta H_{\mathrm{P}} \cdot m}
$$

where $\mathrm{d} H / \mathrm{d} t$ is the heat flow measured by the DSC, $\mathrm{m}$ is the mass of pure HDDA monomer in the sample, $\Delta H_{\mathrm{P}}$ is the experimentally determined enthalpy of polymerization for the acrylate double bond, confirmed by the supplier $\left(\Delta H_{\mathrm{P}}=684 \mathrm{~kJ} / \mathrm{kg}\right)[11,12]$. The rate calculations represent the number fraction of $\mathrm{C}=\mathrm{C}$ bonds reacting in the sample per unit exposure dose. The percent conversion $\alpha$ is then calculated by summing the exothermic heat flow up to a time $t$, and dividing by the expected heat of polymerization of HDDA:

$$
\alpha(t)=\frac{\Delta H_{\text {sample }}(t)}{\Delta H_{\mathrm{P}} \cdot m} \times 100
$$

\subsection{Comparing real time FTIR and photoDSC}

Photopolymerization results, percent conversion $\alpha(t)$ with respect to UV exposure dose, from the three ceramic suspensions are shown in Fig. 8, along with photoDSC results for comparison. The data points obtained by the real time FTIR experiments show good agreement with the photoDSC data for all four samples. Photopolymerization rate $\left(R_{\mathrm{P}}\right)$ per UV exposure dose, calculated from the photoDSC data, is presented in Fig. 9. Although $R_{\mathrm{P}}$ from the RTFTIR data could not be calculated, one would expect the same trends, based on the agreement shown in the conversion $(\alpha(t))$ data. In general, the conversion rate is initially fast, until a 'gel point' is reached, at which the newly polymerized chains begin to inhibit monomer mobility. The maximum photopolymerization rate, $R_{\mathrm{P}}$, may then be associated with the so-defined 'gel point.' The plateaulike region well beyond the 'gel point' seems to confirm this, as the monomers become 'frozen in' and unable 


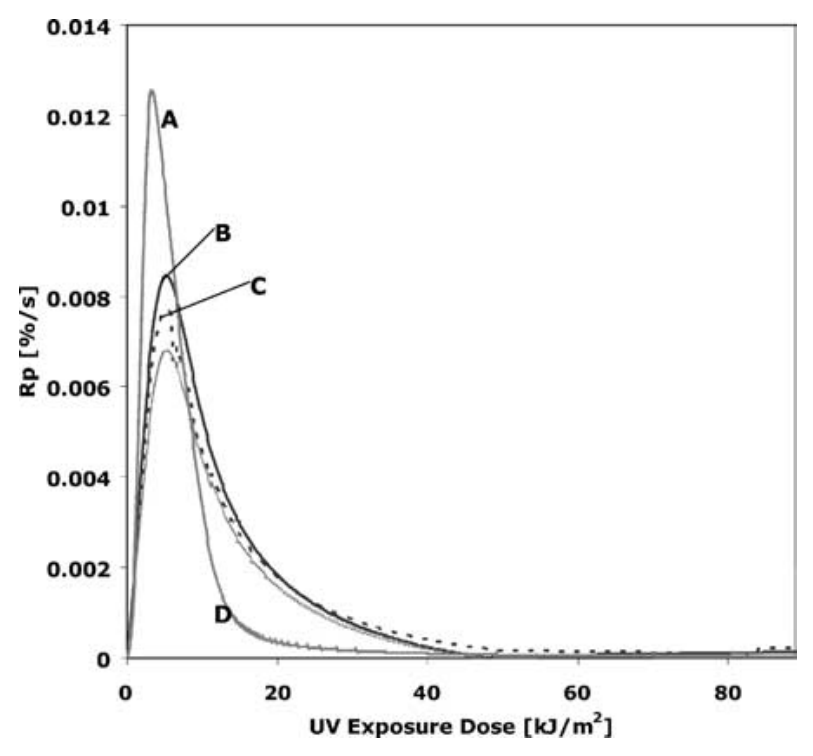

Figure 9 Photo DSC $R_{\mathrm{p}}$ vs. UV exposure dose.

to polymerize fully to $100 \%$. This is most apparent in the data set of pure HDDA monomer, which shows a maximum converision of $57 \%$, lower than that of all the suspensions with alumina, although the initial rate of photopolymerization was much higher than those of the alumina suspensions. In the alumina suspensions, the type and concentration of photoinitiator present was shown to affect the reaction rates. The combination of $1 \% \mathrm{PPO}$ and $1 \% \mathrm{HK}$ accelerated the polymerization reaction, indicated by the maximum value of $R_{\mathrm{P}}$ in Fig. 9, and allowed for higher final conversion over the same suspensions containing $2 \% \mathrm{HK}$ and $0.5 \% \mathrm{HK}$, respectfully. The addition of $\mathrm{PPO}$, which is activated at slightly higher wavelengths of ultraviolet radiation than HK (Fig. 2), increases the concentration of active photoinitiators within the suspension when the UV source is turned on at $t=0$. Essentially, the addition of PPO and HK enables a wider range of the UV source-emitted wavelengths to initiate photopolymerization. Based on these results, in ceramic stereolithography, which utilizes a $325 \mathrm{~nm}$ UV laser, the combination of PPO and HK should prove superior in terms of photopolymerization rate and final conversion.

\subsection{Layered FTIR analysis}

In FTIR experiments, the transmitted IR beam going to the detector contains information over the average thickness of the sample. In photocuring thin films, one would expect that the outermost layer directly exposed to the UV source to have a higher conversion than those beneath it at any given exposure dose. This preferential surface curing behavior is important in photocurable suspensions containing a large amount of scattering and absorbing particles, such as ceramic and photoinitiators. The results of this experiment for the alumina resin A, containing both PPO and HK, shown in Fig. 10, show the FTIR determined percent conversions for each of the three layers at various UV exposure doses and their computed average percent conversion. For comparison, the RTFTIR curve is also shown. As expected,

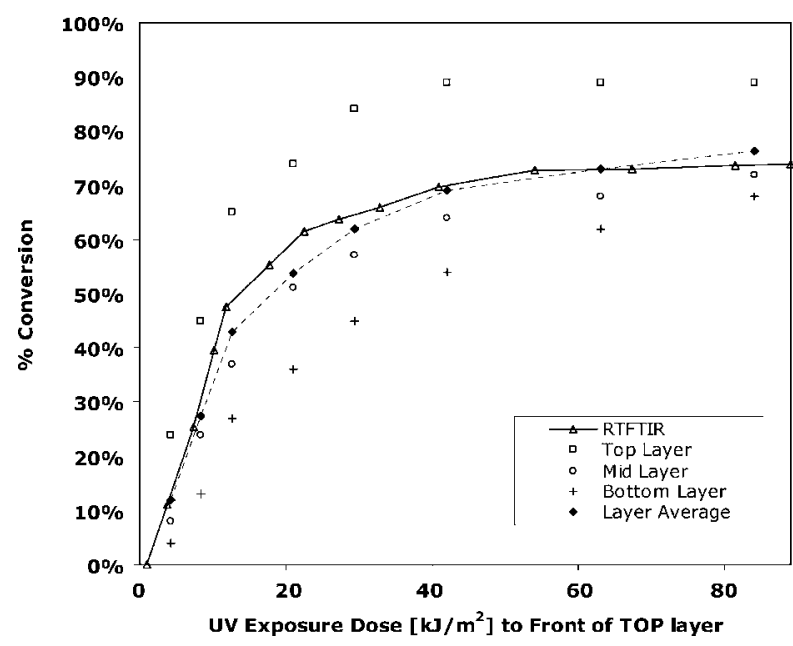

Figure $10 \%$ Conversion vs.Exposure dose in layered FTIR.

the top layer exhibits fastest photopolymerization rate and highest percent conversion, over the mid and bottom layers. The effects of scattering by the alumina particles and absorption by the photoinitiator species act to decrease the photon concentration as a function of distance through the sample, leading to slower polymerization rates and lower final conversion of the bottom layer. The determined average conversion of the stacked layers shows good agreement with the previously obtained RTFTIR data. This highlights the fact that in photopolymerizable suspensions containing scattering and absorbing centers, FTIR will only quantify an average value over the thickness of the sample. Furthermore, future RTFTIR experiments should consider the effect of photon pathlength capability and its impact on photopolymerizing behavior through the sample.

This layered FTIR study also proves useful when comparing with the photopolymerization inside an actual stereolithography machine (SLA 250/40). This is illustrated in Fig. 11. When roughly comparing the RTFTIR data of the suspension containing both PPO and HK to an actual ceramic stereolithographyfabricated piece from the same suspension, in order to photopolymerize a flat piece having a thickness of

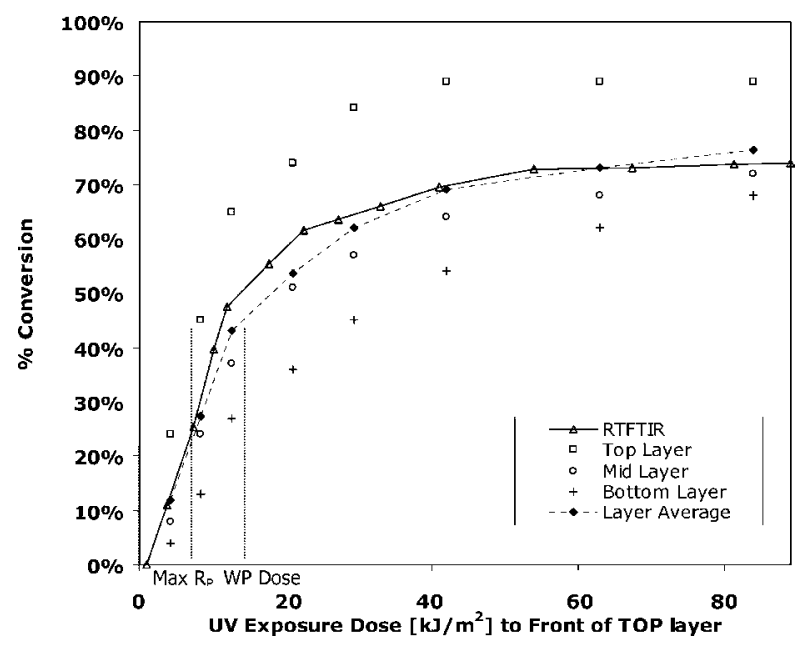

Figure 11 Comparing stereolithography cured pane with FTIR conversion data. 
150 microns on the SLA 250/40 (3D Systems), a dose of $15 \mathrm{~kJ} / \mathrm{m}^{2}$ was required. Note that this is not a rigid comparison because the UV source used in this FTIR experiment emits at several wavelengths over the shortrange UV spectrum (facilitating the activation of photoinitator species), whereas the UV laser in the SLA 250/40 has a monochromatic output at $325 \mathrm{~nm}$. On the percent conversion vs. dose, this dose corresponds to a percent conversion just beyond the expected 'gel-point' of the resin.

\section{Conclusion}

The purpose of these experiments was to develop and utilize an FTIR-based method in characterizing the photopolymerization reaction of photocurable ceramic suspensions used in ceramic stereolithography. Ultraviolet-photocurable alumina suspensions at 50 vol\% loading were created in 1,6-hexanedioldiacrylate monomer (HDDA) with different types and concentrations of photoinitiators. Real-time FTIR (RTFTIR) experiments consisted of a thin film of suspension placed in the IR transmission path while exposed to a constant UV source. With increasing UV exposure dose, the IR scans revealed a decrease in $\mathrm{C}=\mathrm{C}$ double bond peak area, which was then converted into a monomeric percent conversion (to polymer). The Real Time FTIR experiment proved to be feasible and also showed excellent agreement with photopolymerization data from photoDSC experiments, performed on the same alumina-HDDA suspensions.

A layered method of FTIR, was also developed, in which multiple layers of a suspension, after being exposed to a certain UV dose at the top layer, could be separated and analyzed independently. Results show that photopolymerization rate and percent conversion scales with distance through the sample, indicating that scattering and absorption effects through the uppermost layers serve to decrease the photopolymerization reaction at the bottom levels.

\section{References}

1. C. HOY LE, "Radiation Curing: Science and Technology" (Plenum Press, New York, 1992) p. 61.

2. M. L. GRiffith and J. W. Halloran, J. Amer. Ceram. Soc. 79 (1996) 2601.

3. T. CHARTIER, R. PENARROYA, C. PAGNOUX and J. F. B A UMARD, J. Eur. Ceram. Soc. 17 (1997) 765.

4. R. GARG, R. K. PRUD' HOMME and I. A. AKSAY, J. Opt. Soc. Am. A. 15 (1998) 932.

5. H. LiAO and T. COYle, J. Canadian Ceram. Soc. 65 (1996) 254.

6. M. L. GRiffith and J. W. Halloran, J. Appl. Phys. 81 (1997) 2538.

7. C.-H. CHANG, A. MAR, A. TIEFENTHALER and D. WOSTRATZKY, in "Handbook of Coatings Additives" (Marcel Dekker, New York, 1992) Vol. 2, p. 145

8. K. S. ANSETH, C. N. BOWMAN and N. A. PEPPAS, J. Polym. Sci., Part A: Polym. Chem. 32 (1994) 139.

9. J. G. Kloosterboer, G. M. M. VAN DE HEI, R. G. GOS SINK and G. C. M. DORTANT, Polym. Commun. 25 (1984) 322.

10. C. LOWE, "Radiation Curing in Polymer Science and Technology" (Elsevier, New York, 1993) Vol. 4, p. 92.

11. G. A. BRADY and J. W. HALLORAN, J. Mater. Sci. 33 (1998) 4551.

12. G. A. BRADY, T.-M. CHU and J. W. HALLORAN, in Solid Free Form Fabrication Proceedings, Austin, 1996, edited by J. J. Beaman, J. W. Barlow, D. L. Bourell and R. H. Crawford (SFF Symposium, Austin, 1996) p. 403.

Received 27 February

and accepted 12 August 2004 